\title{
Simulation of the Electrons' Motion in a Segmented Hollow Cathode Discharge: Effects of Magnetic Field, Number of Electrode Segments and Cathode - Anode Surface Ratio
}

\author{
Non-member Zoltán Donkó \\ (Research Institute for Solid State Physics, Hungary) \\ Non-member \\ Károly Rózsa \\ (Research Institute for Solid State Physics, Hungary) \\ Non-member \\ Roderick C. Tobin \\ (Dept. of Physics, Monash University, Australia)
}

\begin{abstract}
We have investigated the motion of electrons in a new, high voltage segmented hollow cathode discharge, applicable as an efficient pumping source for charge transfer excited UV metal ion lasers. We have used Monte Carlo simulation of the electrons' motion to obtain the spatial distribution of ion production, electron energy distributions, and the fraction of oscillating electrons in the discharge. We present the results of an analysis of the effects of the longitudinal magnetic field, the number of electrode segments and the cathode anode surface ratio. The results of the simulations explain the experimentally observed behaviour of the investigated discharges. Our study with a longitudinal magnetic field confirms the existence of oscillating electrons even at relatively high pressures.
\end{abstract}

Keywords: Hollow Cathode Discharges and Lasers, Monte Carlo Simulation

\section{Introduction}

High voltage hollow cathode discharges provide higher electron energy for the excitation mechanism of lasers and exhibit improved discharge stability compared to conventional (e.g. cylindrical) hollow cathode discharges. ${ }^{(1,2)}$ The higher operating voltage $(600-1200 \mathrm{~V})$ also provides higher metal vapor concentration because of the increased sputtering of the cathode due to the bombardment by more energetic ions. The latest development in the design of 'laser purpose' hollow cathode discharges is the segmented hollow cathode (SHC) discharge ${ }^{(3)}$, shown in Fig. 1, for two different number of electrode segments (4 and 6). In the following we identify these discharges as $\mathrm{SHC}-4$ and $\mathrm{SHC}-6$, respectively. In the SHC discharges the working surfaces of the cathode and anode electrodes are situated on a common cylindrical surface. Using the SHC configuration all the metal ion laser transitions investigated have shown higher gain over earlier constructions. ${ }^{(2-5)}$

The effect of magnetic field and the different 'geometrical' modifications of the discharge are important in the understanding of the discharge and its optimization for UV (and possible VUV) laser purposes.

The SHC discharge is characterized by a positive - slope voltage - current curve, as it can be seen in Fig. 2(a) for $p=7.5$ mbar buffer gas ( $\mathrm{He}+4 \% \mathrm{Ar}$ ) pressure, for the $\mathrm{SHC}-4$ and $\mathrm{SHC}-6$ discharges. ${ }^{(4)}$ (The data for the discharge with six segments are taken from a slightly different discharge arrangement, namely a hol- low-anode-cathode discharge having three anodes. ${ }^{(4)}$ In the HAC construction thin slotted cylindrical anodes are placed concentrically within a cylindrical cathode cavity, while in the segmented tube the anodes and cathodes together form the cylindrical cavity.)

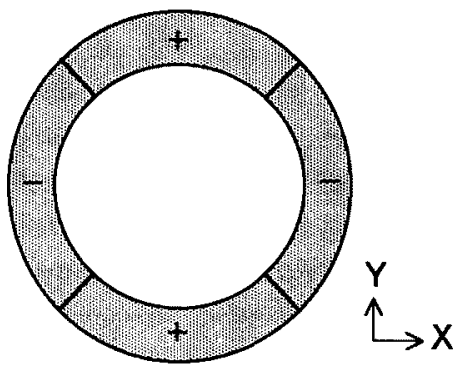

(a)

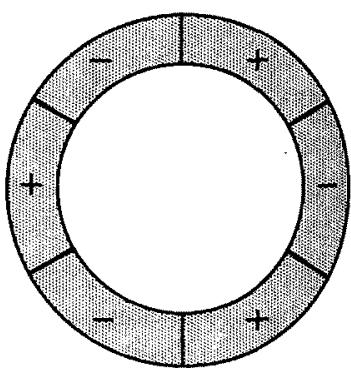

(b)
Fig. 1. The cross section of the segmented hollow cathode discharge having (a) 4 and (b) 6 electrode segments. The electrodes are insulated from each other using thin ceramic spacers (not indicated on the figure).

In conventional hollow cathode discharges the application of longitudinal magnetic field lowers the discharge voltage at constant current. An opposite effect was observed in a slotted hollow-anode-cathode (HAC) discharge ${ }^{(6)}$ and in a segmented hollow cathode with four segments. ${ }^{(4)}$ In each case the magnetic field increased the discharge 
voltage and thus improved the performance of the investigated $\mathrm{Cu}-$ II $780.8 \mathrm{~nm}$ lasers. Fig. 2(b) shows the experimentally observed dependence of the discharge voltage on the longitudinal magnetic field at constant discharge current. ${ }^{(4)}$ We have chosen the 0 to $20 \mathrm{mT}$ range of the longitudinal magnetic field, as the most interesting range for our study. At higher magnetic field the electrons start to return to that cathode which they were launched from, and the voltage always decreases (a known effect in hollow cathode discharges).
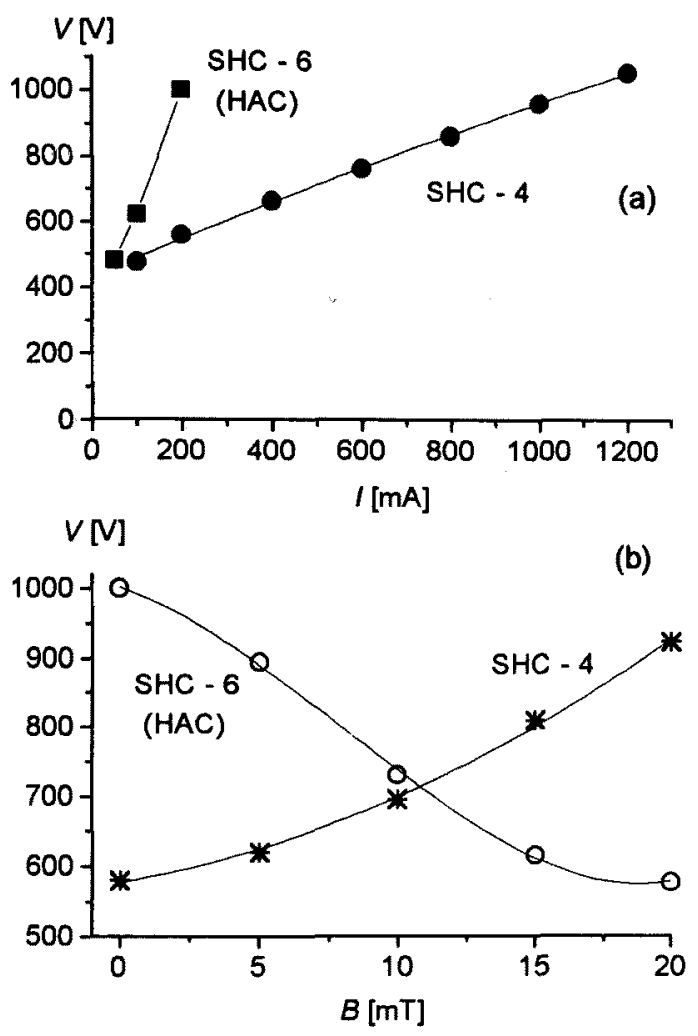

Fig.2. (a) The voltage - current characteristics of segmented hollow cathode discharges having 4 (SHC -4$)(\bullet)$ and 6 electrode segments (SHC -6) (D), at $p=7.5 \mathrm{mbar}$ buffer gas $(\mathrm{He}+4 \% \mathrm{Ar}$ ) pressure. (b) The dependence of the discharge voltage $V$ on the applied longitudinal magnetic field $B$ at constant discharge current of $I=200 \mathrm{~mA}$ (corresponding to a current density $j=67 \mathrm{~mA} / \mathrm{cm}^{2}$ in a $4 \mathrm{~mm}$ diameter, $5 \mathrm{~cm}$ long discharge tube $\left.{ }^{(4)}\right)$, at $p=7.5 \mathrm{mbar}$, for the SHC $-4(*)$ and SHC $-6(\mathrm{O})$ discharges. ${ }^{(4)}$

The cathode - anode surface ratio has also been found to have a significant effect on the voltage-current characteristics and the laser performance (gain) of the SHC discharge. The decreasing cathode area resulted in an increased discharge voltage and enhanced gain on the $\mathrm{Cu}-$ II $780.8 \mathrm{~nm}$ infrared transition. $(7,8)$

We have applied Monte Carlo simulation to trace the trajectories of electrons in the SHC discharge. ${ }^{(9)}$ In this paper we present several discharge characteristics, obtained from the simulations: the spatial distribution of the ionization source, the energy distribution of ionizing electrons and electrons absorbed by the anode, the fraction of the oscillating electrons in the discharge, and the statistics of electron avalanches. The effect of the longitudinal magnetic field and the effect of modifications of the discharge arrangement (varying the number of electrode segments and the cathode - anode surface ratio) are investigated on selected discharge characteristics (the spatial distribution on the ionization source, the energy distribution of electrons absorbed by the anode, and the fraction of the oscillating electrons in the discharge).

Our simulation of the electrons' motion in the SHC discharge can be considered as the first step towards the self-consistent modeling of this discharge. As a result of the present simulations we obtained detailed information about the motion of electrons in the SHC discharge, which allows us to explain some of the basic features of the discharge.

In Section 2 of the paper we present the method of the simulation and summarize the basic assumptions of our model. (A more detailed description may be found in Ref. 10.) The results are presented and discussed in Section 3. The effect of the magnetic field is presented in Section 3.1 and 3.2 for the discharges with 4 and with 6 segments, respectively. The effect of the varying cathode - anode surface ratio is given in Section 3.3. Section 4 summarizes the results.

\section{The simulation method and model}

In the presence of an electromagnetic field the trajectory of a particle can be traced by the integration of the equation of motion:

$$
m \frac{d^{2} \mathbf{r}}{d t^{2}}=q[\mathbf{E}+\mathbf{v} \times \mathbf{B}]
$$

where $m$ and $q$ are the mass and charge of the particle, $\mathbf{r}$ and $\mathbf{v}$ are the particle's position and velocity vectors, $\mathbf{E}$ and $B$ are the electric and magnetic field vectors.

In Monte Carlo (MC) simulations the free path of the particle along the trajectory is obtained by solving the following equation, see e.g. Ref. 11:

$$
\int_{s_{0}}^{s_{1}} n \sigma[\varepsilon(s)] d s=-\ln \left(1-R_{01}\right)
$$

where $s_{0}$ is the initial position of the particle, $s_{1}$ is the position of the next collision on the curvilinear coordinate $s, n$ is the gas density, $\sigma$ is the sum of the cross sections of all possible collision processes, $\varepsilon$ is the kinetic energy of the particle, and $R_{01}$ is a random number uniformly distributed on the $[0,1)$ interval. In the general case $(|\mathbf{E}| \neq 0)$, equations (1) and (2) have to be integrated numerically. In the case of electric field free regions (1) is simplified, as $\varepsilon$ and thus $\sigma$ does not change between collisions. Thus integration of (2) can be carried out analytically and $s_{1}$ can be easily calculated. Between collisions the particles moves either on a straight trajectory $(|\mathbf{B}|=0)$, or on a spiral trajectory $(|\mathbf{B}| \neq 0)$ with radius:

$$
\rho=\frac{m \mathrm{v}_{0}}{q B}
$$

where $\mathrm{v}_{0}$ is the initial speed of the particle and $B=|\mathbf{B}|$. 
The basic assumptions and simplifications of the model are summarized as follows:

1. In the case of the segmented hollow cathode discharge, there is no data available for the length of the cathode sheath, and near the edges of the cathodes a tangential electric field of unknown spatial dependence appears. Thus we assume that the cathode dark space is very narrow (collisionless). This major simplifying assumption of the model is justified by the very narrow cathode sheath at high current densities. ${ }^{(12)}$

2. In our simulations the electrons are launched from the cathode with a kinetic energy corresponding to the full cathode fall voltage added to an average energy of ejected electrons (for which we use $10 \mathrm{eV}$ ). This way the electrons are monoenergetic when they enter the negative glow. The negative glow is free of electric field, what makes the simulation fast enough to calculate relatively 'noise-free' 2-dimensional ionization source functions.

3. We present our simulation results for a helium discharge. The discharge parameters (pressure, voltage, current density) originate from experimental studies of infrared $(\lambda=780.8 \mathrm{~nm})$ cathode-sputtered copper ion laser. $(4,7,8)$ The laser used a buffer gas of helium with $4-6 \%$ admixed argon to enhance cathode sputtering. We believe that the effects investigated (focusing property of the SHC electrode arrangement, effect of magnetic field on the discharge parameters,...) are relatively insensitive to the small admixed amount of argon.

4. We assume that the discharge is homogeneous in the $z$ direction (which is perpendicular to the $x-y$ plane). The electrons were started from uniformly distributed random positions on the cathode surface, at $z=0$. Their initial velocity vector was normal to the cathode surface.

5. The elementary processes considered in our model are anisotropic elastic scattering of electrons from He atoms, electron impact excitation and ionization of $\mathrm{He}$ atoms (source of cross sections: Refs 11,13,14).

6. The electrons for which the trajectories arrive at the cathode surface were treated according to their energy. Electrons with the full cathode fall potential (being able to reach the cathode surface after slowing down in the opposing field of the cathode sheath) were reflected from the cathode with a probability of $R=0.2 .^{(15)}$ Electrons with less energy (not being able to traverse the cathode sheath) were always reflected from the zero-thickness sheath. The anode surfaces were assumed to be perfectly absorbing. The electrons were traced in the simulation until they were absorbed by one of the electrodes or their energy fell below the ionization potential of He (except when calculating electron energy distributions).

Our model, as it is the first approach to investigate the properties of the SHC discharge, is not a self-consistent model. We assumed the cathode fall voltage (values taken from experiments) to drop in an infinitely narrow cathode sheath. This sheath can, however, reflect the electrons approaching the cathode, thus we include the reflection and oscillation of fast electrons - one major reason of the hollow cathode effect - in our simulation.
The Monte Carlo simulation provides a simple means of the determination of the examined characteristics by accumulating the data during the simulations (e.g. the spatial distribution of the ion production is obtained by storing the position of each ionization collision). The determination of the fraction of the oscillating electrons depends on the definition of the term 'oscillating electron'. The presence of oscillating electrons is one of the reasons for the existence of the hollow cathode effect. The oscillating electrons may traverse the space several times between the opposite cathode surfaces of a hollow cathode discharge.$^{(16-18)}$ We have found, that apart from the oscillations of primary electrons between the opposing cathodes, the secondary electrons (created in ionizations) further contribute to the hollow cathode effect, if they are reflected from the opposing cathode surfaces. This type of reflection occurs only in hollow cathode type arrangements. Thus we have defined the fraction $(\eta)$ of oscillating (and reflected) electrons in the following way: launching one electron from one of the cathodes, we calculated the number of electrons reflected from the opposing cathode or cathode sheath (in the given electron avalanche). We obtained $\eta$ as the ratio of the number of reflected electrons to the total number of electrons participating in the given avalanche, averaged over a large number of avalanches.

The number of primary electrons in the simulations was typically $10^{5}$, while for the calculation of the two dimensional ionization source functions it was $10^{6}$. Including all the electrons created in ionizing collisions, we traced about $10^{7}$ electrons to obtain each of the presented 2 -dimensional ionization source functions.

\section{Results and Discussion}

In Section 1 we gave some information about the change of the discharge voltage (at constant current) as a result of the application of the longitudinal magnetic field and as a result of the changing cathode - anode surface ratio. As the discharge voltage and current mutually define each other (see Fig. 2(a)), it is also possible to study the discharge at fixed voltage and then the discharge current is subject to change due to the influence of the magnetic field or other effects. The electron energy distributions, spatial distribution of ion production and the fraction of oscillating electrons depend on the longitudinal magnetic field, the discharge geometry and also on the discharge voltage. In order to illustrate the effect of the magnetic field and the discharge geometry we have kept the voltage constant in our calculations. Studying the discharge at fixed voltage also has the advantage that the possible maximum electron energy is constant, thus the energy distributions obtained in different cases can directly be compared.

All the results are given for a bore diameter $D=4 \mathrm{~mm}$. In the following we present the results obtained for the magnetic field free case, for discharges having 4 and 6 electrode segments. The spatial distribution of the ionization source function $S^{+}(x, y)$ is displayed in Fig. 3(a), for a SHC -4 discharge, for current of $I=200 \mathrm{~mA}$ (corresponding to $j=67 \mathrm{~mA} / \mathrm{cm}^{2}$ current density in a $5 \mathrm{~cm}$ long discharge tube $\left.{ }^{(4)}\right)$ and $p=7.5$ mbar. 


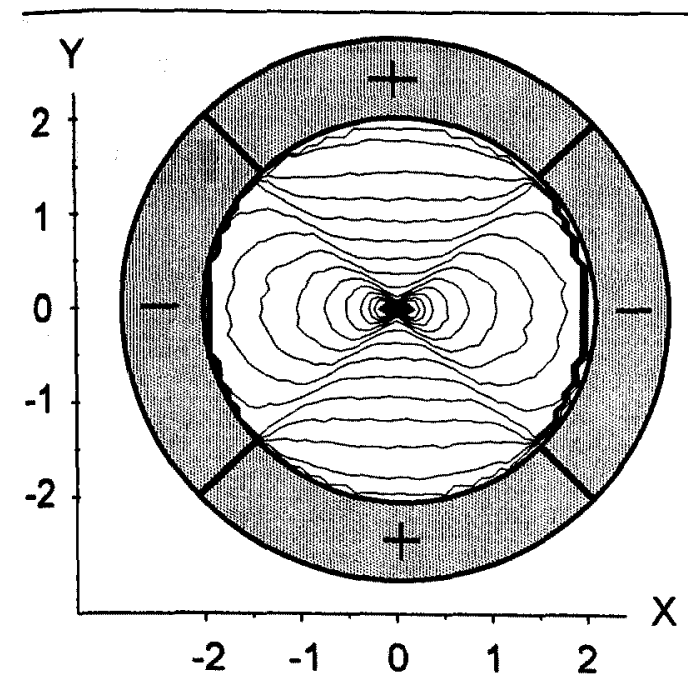

(a)
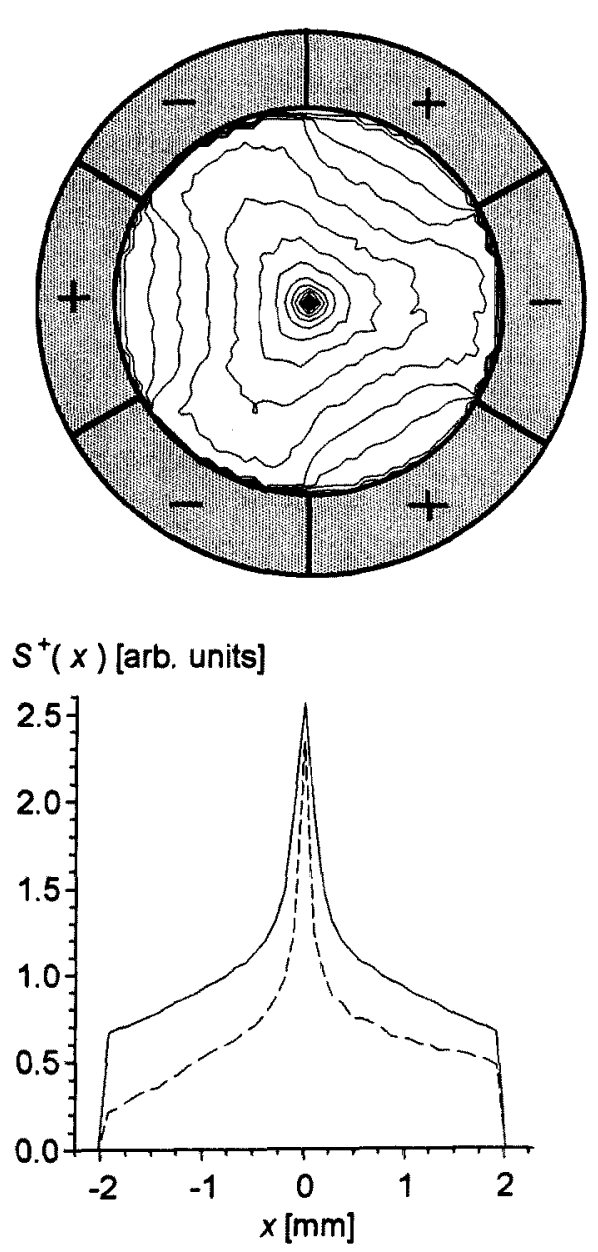

Fig. 3.(a,b) The $S^{+}(x, y)$ spatial distribution of the ion production in the cross section of a segmented hollow cathode discharge of $4 \mathrm{~mm}$ diameter. $S^{+}(x, y)$ is normalized as given by eq.(4), $x$ and $y$ are given in $\mathrm{mm}$ (the scale is the same in the forthcoming figures). The discharge parameters are: (a) $V=580 \mathrm{~V}$ and $p=7.5 \mathrm{mbar}$ for the SHC -4 discharge and (b) $V=1000 \mathrm{~V}$ and $p=7.5 \mathrm{mbar}$ for the SHC -6 discharge. The contour step corresponds to 0.1 change in the $S^{+}(x, y)$ function. (c) The source of ionization along the X axis, $S^{+}(x)$, for the SHC $-4(-)$ and SHC $-6(--)$ discharges. $S^{+}(x)$ is obtained from ionization events in the range: $-0.05<y<0.05$, and is given in arbitrary units.

Fig. 3(b) shows $S^{+}(x, y)$ for a SHC - 6 discharge, for the same $(I=200 \mathrm{~mA})$ current and pressure ( $p=7.5$ mbar). The discharge voltage is $V=580 \mathrm{~V}$ for the SHC -4 and $V=1000 \mathrm{~V}$ for the SHC -6 .

The $S^{+}(x, y)$ functions plotted in Fig. 3 (and in the following figures) are normalized as

$$
\iint S^{+}(x, y) d x d y=M
$$

where $M$ is the multiplication (the average number of electrons participating in an electron avalanche).

It can be seen in Fig. 3(a,b) that the ionization is sharply peaked in the centre of the hollow cathode, due to the focusing effect of the curved cathode surfaces, which is still significant even at the relatively high pressure of 7.5 mbar. Fig. 3(c) shows the cut of the $S^{+}(x, y)$ functions (plotted in Fig. 3(a,b)), along the $X$ axis $(y=0)$. This corresponds to the line between the midpoint of the cathodes in the SHC -4 discharge and the line between the midpoint of one of the anodes $(x=-2.5 \mathrm{~mm})$ and one of the cathodes $(x=2.5 \mathrm{~mm})$ for the SHC -6 discharge. Consequently, the curve for the $\mathrm{SHC}-4$ is symmetric and the curve for the SHC - 6 is asymmetric and also shows the ion production near the anode.

The energy distribution of electrons absorbed by the anode is plotted in Fig. 4(a) for the SHC -4 discharge, for $p=7.5$ mbar, $B=0, V=580 \mathrm{~V}$. (This distribution was obtained by storing the kinetic energy of each individual electron absorbed on the anode surface.) The high operating voltage of the segmented hollow cathode discharge can be partly explained by the loss of these high-energy electrons, which could have produced additional ionization if they had been reflected from the cathode or cathode sheath. ${ }^{(19)}$

The spike - like structure of the electron energy distribution function at high energies, plotted in Fig. 4(a) (and in the following figures) is caused by the possible values of electron energy loss in collision processes. The energy loss of electrons occurs in the inelastic processes, where the possible energy loss is between the first excitation level and the ionization potential of helium. The peak at the highest electron energy represent those electrons which did not participate in any inelastic processes along their trajectory from the cathode to the anode and have the whole energy corresponding to the cathode fall voltage. The next peak towards lower energy corresponds to those electrons which have participated in one inelastic collision. The peaks at lower energies are gradually smoothed by the $\approx 5 \mathrm{eV}$ wide range of the possible energy loss and the random initial energy of electrons created in ionizations.

The cross section of electron impact ionization of helium (displayed in Fig. 4(b)) peaks at $\varepsilon_{\max } \approx 120 \mathrm{eV}$. In the investigated discharges the electrons may acquire much higher energies than $\varepsilon_{\max }$. The $f^{+}(\varepsilon)$ energy distribution of ionizing electrons is also plotted in Fig. 4(b), for $p=7.5$ mbar, $B=0, V=580 \mathrm{~V}$. (The $f^{+}(\varepsilon)$ distribution was obtained by accumulating data about individual ionization processes in the course of the simulation.) It is noted that approximately $50 \%$ of the ions are created by electrons having an energy $\varepsilon>\varepsilon_{\max }$, meaning that the high-energy 
electrons play an important role in the ionization in the discharge.
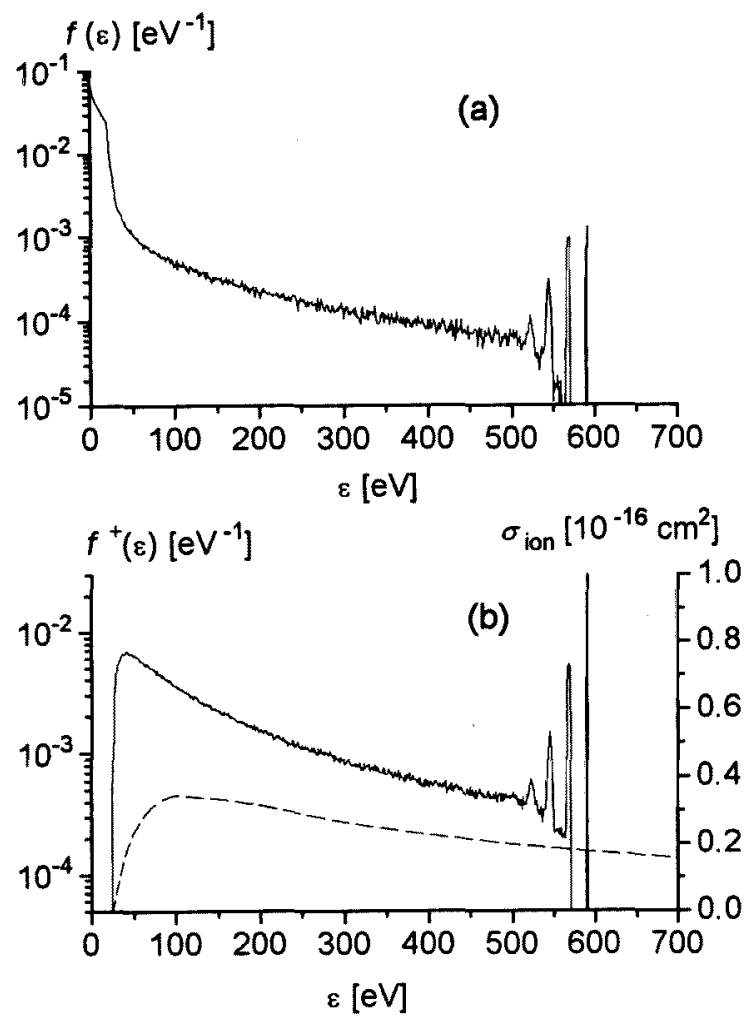

Fig. 4.(a) The $f(\varepsilon)$ energy distribution of electrons absorbed by the anode, for $p=7.5 \mathrm{mbar}, B=0, V=580 \mathrm{~V}$. Note the high number of absorbed high-energy electrons. (b) The $f^{+}(\varepsilon)$ energy distribution of ionizing electrons (—, left scale) for $p=7.5 \mathrm{mbar}, B=0, V=580 \mathrm{~V}$ and the $\sigma_{\text {ion }}$ cross section of electron impact ionization ${ }^{(13)}$ of helium (- - , right scale). The cross section peaks at approximately $\varepsilon_{\max } \approx 120 \mathrm{eV}$.

The electron energy distributions shown in Fig. 4(a,b) (and in the following figures) are normalized according to:

$$
\int f(\varepsilon) d \varepsilon=1
$$

Fig. 5(a) displays the results of the calculations for the fraction of oscillating (and reflected) electrons, $\eta$. The fraction of oscillating electrons decreases with increasing pressure, as it can be seen in Fig. 5(a) (for $B=0$ ).

The number of ionizations in subsequent electron avalanches (initiated by the emission of one single electron from the cathode) is different. Counting the number of avalanches in which $k$ ions were created, we can obtain the $P(k)$ probability distribution. The $P(k)$ distribution for $p=7.5$ mbar, $B=0, V=580 \mathrm{~V}$ is displayed in Fig. 5(b). It can be seen in Fig. 5(b) that there is a significant number of electron avalanches, in which no ions are created $(k=0)$, i.e. the primary electron emitted from the cathode is absorbed by the anode without making any ionizations. The probability of this is denoted by $P_{0}$. The most probable number of ions created in an electron avalanche (for the above discharge parameters) is $k=10$.
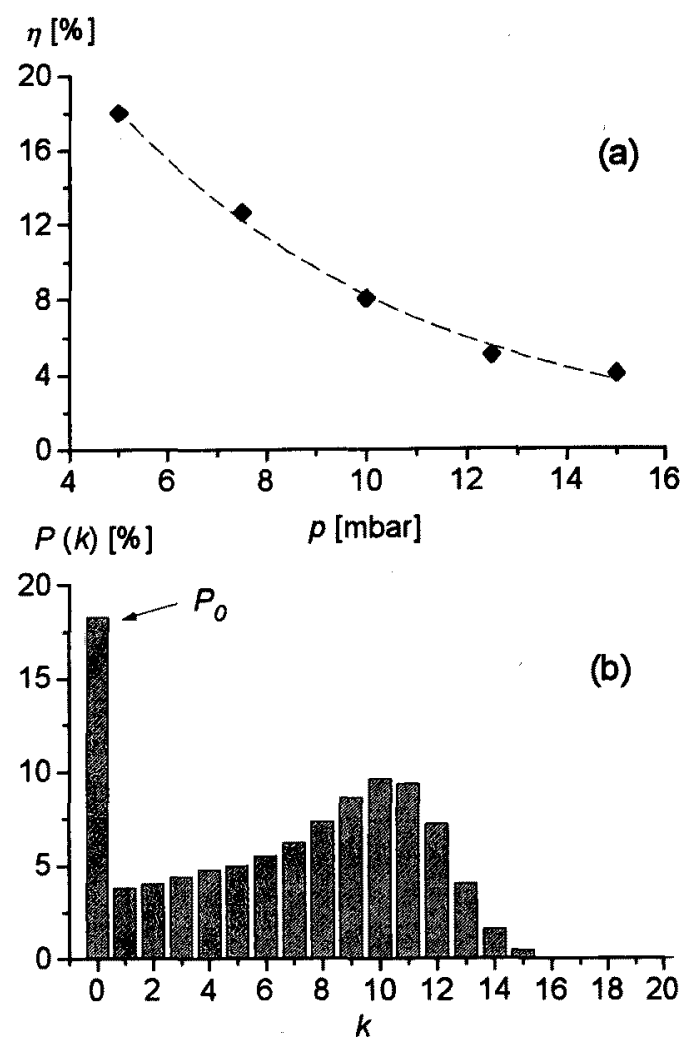

Fig. 5. (a) The $\eta$ fraction of oscillating electrons in the SHC -4 discharge as a function of the buffer gas pressure $(V=580 \mathrm{~V}, B=0$ ). (b) The $P(k)$ probability distribution of the number of ionizations in an electron avalanche, for $p=7.5 \mathrm{mbar}, B=0, V=580 \mathrm{~V}$. Note the high probability $\left(P_{0} \approx 18 \%\right.$ ) of a primary electron being absorbed by the anode without making any ionizations.

In the following we present the simulation results of the effect of longitudinal magnetic field (on the $\mathrm{SHC}-4$ and SHC -6 discharges) and the changing of the cathode-anode surface ratio on selected discharge characteristics.

\subsection{The effect of magnetic field on the SHC -4}

In this section we present the results obtained for the effect of the longitudinal magnetic field on a discharge having four electrode segments. The spatial distribution of the ionization source function $S^{+}(x, y)$ is displayed in Fig. 6 , for $V=580 \mathrm{~V}$ discharge voltage and $p=7.5 \mathrm{mbar}$, for magnetic field strengths $B=10 \mathrm{mT}$ and $B=20 \mathrm{mT}$. In the presence of magnetic field (because of the bending of the trajectories) the 'focal point' observed in Fig. 3(a) (which sharply peaks in the centre of the discharge) is split into two regions of high ionization rate.

The energy spectrum of the electrons absorbed by the anode, plotted in Fig. 7, shows an increasing number of high energy electrons with increasing magnetic field. The simulations have been carried out for the same discharge 
parameters as indicated for Fig. 6. The increased number of absorbed high-energy electrons results in a decreased ionization rate, thus a lower current density, in agreement with experimental observations. ${ }^{(4)}$ The necessary ionization rate to keep the current constant as the magnetic field is applied, can only be maintained by an increased discharge voltage.

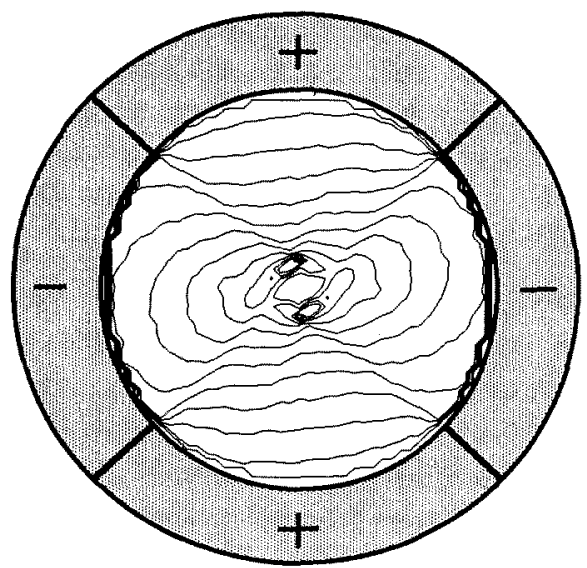

(a)

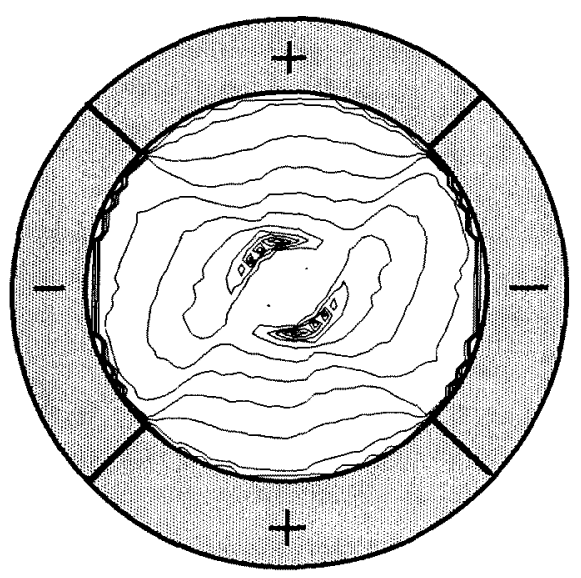

Fig. 6. The effect of magnetic field on the $S^{+}(x, y)$ spatial distribution of the ion production in the cross section of the SHC -4 discharge. The contour step corresponds to 0.1 change in the $S^{+}(x, y)$ function. The discharge parameters are: $V=580 \mathrm{~V}$ and $p=7.5$ mbar. (a) $B=10 \mathrm{mT}$ and (b) $B=20 \mathrm{mT}$

Due to the deflection of the electron trajectories there is also an increasing number of primary electrons, which are absorbed by the anode before making any ionizations. The $P_{0}$ probability of this is shown in Table 1 for different values of the magnetic field. $P_{0}$ increases by approximately $5 \%$ as the magnetic field is increased from $B=0 \mathrm{mT}$ to $B=20 \mathrm{mT}$. Table 1 also includes the data for the $\eta$ fraction of the oscillating (and reflected) electrons. The data shown in Table 1 indicate that the increasing magnetic field reduces the number of oscillating electrons from $12.6 \%$ (at $B=0 \mathrm{mT}$ ) to $9.4 \%$ (at $B=20 \mathrm{mT})$.

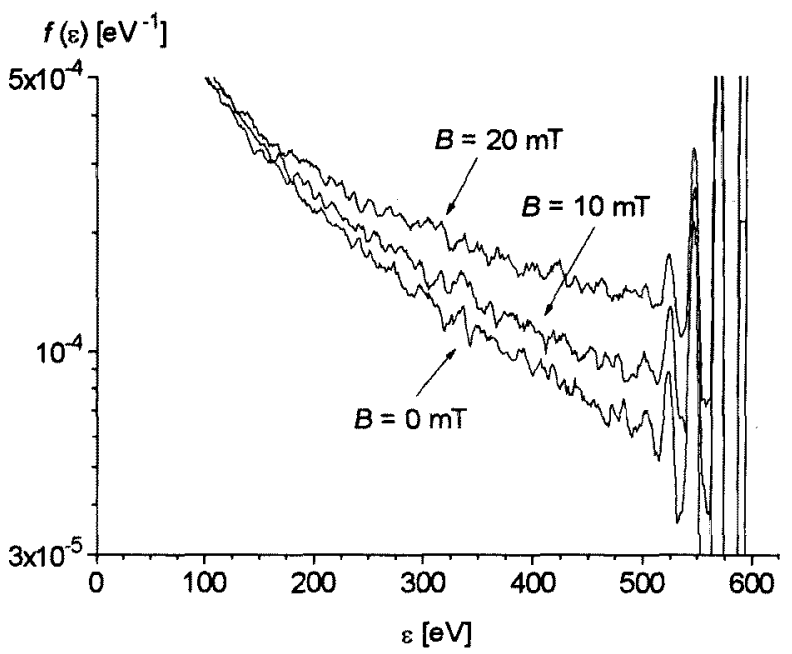

Fig. 7. The high energy part of the $f(\varepsilon)$ energy distribution of electrons absorbed by the anode in the SHC -4 discharge, for $p=7.5$ mbar pressure, $V=580 \mathrm{~V}$ voltage, at different values of the longitudinal magnetic field $B=0$, $B=10 \mathrm{mT}$ and $B=20 \mathrm{mT}$. Note the increased number of high energy electrons as the magnetic field is applied.

Table 1. The effect of the magnetic field on $P_{0}$ and $\eta$ in the SHC -4 discharge $(V=580 \mathrm{~V}, p=7.5 \mathrm{mbar}$ ).

\begin{tabular}{c||c|c}
\hline$B[\mathrm{mT}]$ & $P_{0}[\%]$ & $\eta[\%]$ \\
\hline \hline 0 & 18.3 & 12.6 \\
\hline 10 & 19.9 & 11.6 \\
\hline 20 & 22.8 & 9.4 \\
\hline
\end{tabular}

\subsection{The effect of magnetic field on the SHC - 6}

As it was already illustrated in Fig. 2, the segmented hollow cathode discharge having 6 electrode segments exhibits an opposite behaviour due to the longitudinal magnetic field, compared to the SHC with 4 segments. In this section we present the results for the SHC -6 arrangement, similarly to the SHC -4 in Section 3.1.

The spatial distribution of the ionization source function $S^{+}(x, y)$ is displayed in Fig. 8, for $V=1000 \mathrm{~V}$ discharge voltage and $p=7.5 \mathrm{mbar}$, for magnetic field strengths $B=10 \mathrm{mT}$ and $B=20 \mathrm{mT}$. The ionization source peaks over a broad, roughly cicular area, which increases with the magnetic field. This is in contrast to the sharp peak of the ionization source without a magnetic field, Fig. 3(b).

The energy spectrum of the electrons absorbed by the anode is plotted in Fig. 9. The simulations have been carried out for the following discharge parameters: $V=1000 \mathrm{~V}, \quad p=7.5 \mathrm{mbar}, \quad B=0, \quad B=10 \mathrm{mT}$ and $B=20 \mathrm{mT}$. It can be seen that in the six segment discharge the number of high energy electrons decreases as the magnetic field is applied. This is again in contrast with the case of the four segment discharge, where (at constant voltage) the number of absorbed high energy electrons increased due to the increasing magnetic field, see Fig. 7. The experimentally found decreasing voltage - magnetic field 
curve (Fig. 2(b)) can be partly explained by the reduced number of absorbed high energy electrons.

The increasing magnetic field results in a decreasing number of primary electrons, which are absorbed by the anode before making any ionizations, as indicated by the $P_{0}$ values given in Table 2 . The significantly higher values of $P_{o}$ given in Table 2 for the SHC - 6 discharge, compared to those values for the SHC-4 (see Table 1) are the consequence of the considerably higher discharge voltage. (Note that the cross section of ionization decreases with increasing energy.)

Table 2. also includes the data for the fraction $\eta$ of the oscillating (and reflected) electrons. For the discharge with six segments, the fraction of the oscillating electrons was determined in the following way. The electrons were launched from one of the cathode electrodes. Then $\eta$ was calculated as the ratio of the number of electrons reflected from the other two cathodes or cathode sheaths to the total number of electrons participating in the avalanche, averaged over a large number of avalanches. It can be seen in Table 2 that $\eta$ increases with increasing magnetic field.
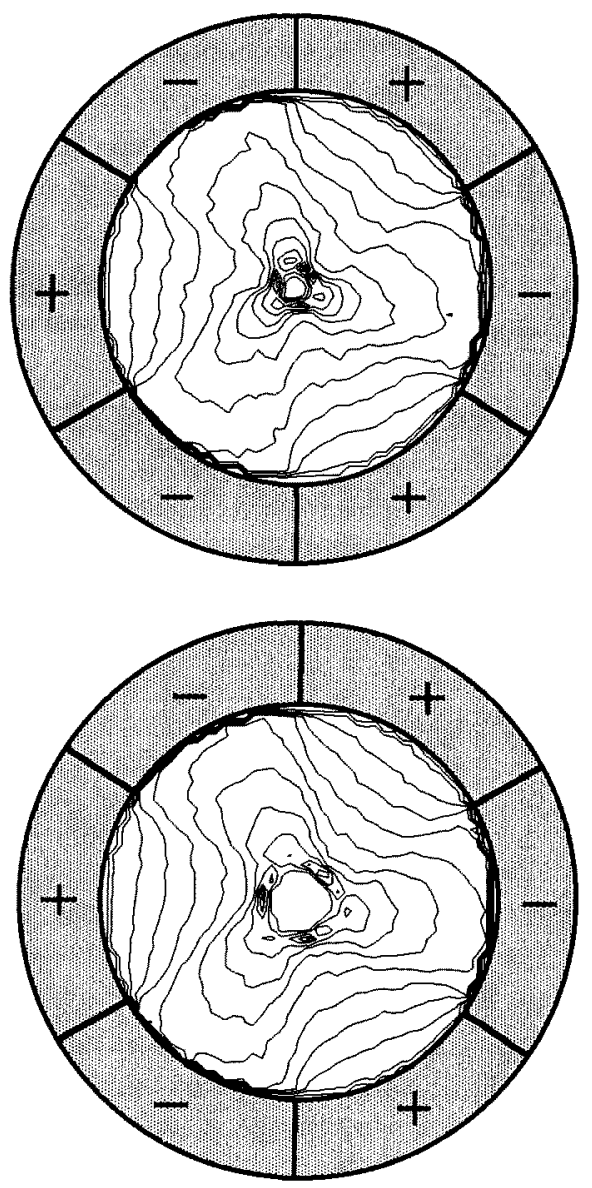

Fig. 8. The effect of magnetic field on the $S^{+}(x, y)$ spatial distribution of the ion production in the SHC -6 discharge. The contour step corresponds to 0.1 change in the $S^{+}(x, y)$ function. The discharge parameters are: $V=1000 \mathrm{~V}$ and $p=7.5$ mbar. (a) $B=10 \mathrm{mT}$ and (b) $B=20 \mathrm{mT}$.

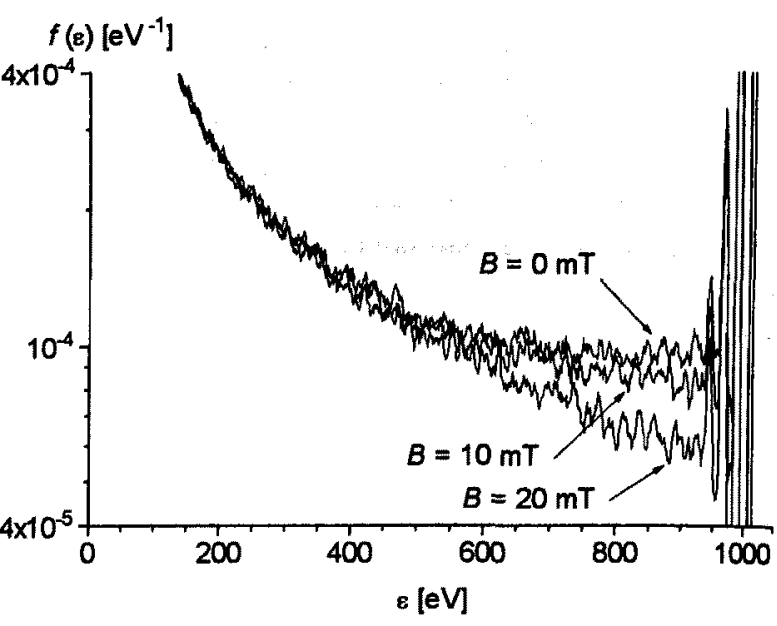

Fig. 9. The high energy part of the $f(\varepsilon)$ energy distribution of electrons absorbed by the anode in the SHC -6 discharge, for $p=7.5 \mathrm{mbar}$ pressure, $V=1000 \mathrm{~V}$ voltage at different values of the longitudinal magnetic field $B=0 \mathrm{mT}, B=10 \mathrm{mT}$ and $B=20 \mathrm{mT}$. Note the decreased number of high energy electrons as the magnetic field is applied.

Table 2. The effect of the magnetic field on $P_{0}$ and $\eta$ in the SHC -6 discharge $(V=1000 \mathrm{~V}, p=7.5 \mathrm{mbar})$.

\begin{tabular}{c||c|c}
\hline$B[\mathrm{mT}]$ & $P_{0}[\%]$ & $\eta[\%]$ \\
\hline \hline 0 & 36.1 & 13.8 \\
\hline 10 & 35.4 & 14.6 \\
\hline 20 & 34.5 & 15.5 \\
\hline
\end{tabular}

In this section we presented the results for the spatial distribution of ion production, the energy distribution of electrons absorbed by the anode, the probability that an electron launched from the cathode is absorbed on the anode without making any ionization, and the fraction of the oscillating electrons in the discharge. The dependence of the latter three important discharge characteristics on the longitudinal magnetic field essentially differs from that of the discharge with four segments, because of the 3 fold symmetry of the SHC -6 discharge. Note that the four segment discharge has opposite, facing cathode electrodes, but in the six segment discharge anodes are opposite to cathodes. It is noted that, if the current is kept constant, the number of oscillating electrons is almost constant regardless of the magnetic field, for both the SHC -4 and SHC -6 discharges. ${ }^{(10)}$ These results confirm that the oscillating electrons play an important role in the ion production.

\subsection{The effect of cathode - anode surface ratio}

A further step in the optimization of the SHC discharge was the investigation on the effect of cathode - anode surface ratio $(C: A)$ on the discharge and laser characteristics. ${ }^{(7,8)}$ In this section we present results for $2: 1$ and $1: 2$ cathode - anode surface ratios. The discharge parameters are taken from experimental studies. ${ }^{(7,8)}$ 
The spatial distribution of the ionization source function $S^{+}(x, y)$ is displayed in Fig. 10, for $V=600 \mathrm{~V}$ discharge voltage and $p=7.5 \mathrm{mbar}$, for cathode - anode surface ratios $C: A=2: 1$ and $C: A=1: 2$.

The energy spectrum of the electrons absorbed by the anode is plotted in Fig. 11, for $V=600 \mathrm{~V}$ discharge voltage and $p=7.5 \mathrm{mbar}$, for different values of the cathode anode surface ratio. Fig. 10 shows an increasing number of high energy electrons, as the cathode - anode surface ratio changes from 2:1 to $1: 2$. This is explained by the increased probability of the absorption of high energy electrons due to the increased absorbing surface. The increased loss of electron energy at lower $C: A$ results in a decreased discharge current at constant voltage (and increased discharge voltage at constant current). ${ }^{(7,8)}$ Due to the increased anode area at $C: A=1: 2$ there is also an increasing number of primary electrons, which are absorbed by the anode before making any ionizations, see the $P_{0}$ values shown in Table 3. Table 3, also includes the data for the $\eta$ fraction of the oscillating (and reflected) electrons. The increasing anode area significantly reduces the fraction of oscillating electrons in the discharge. However, the focusing effect of the concave cathodes is still significant (see Fig. 10).

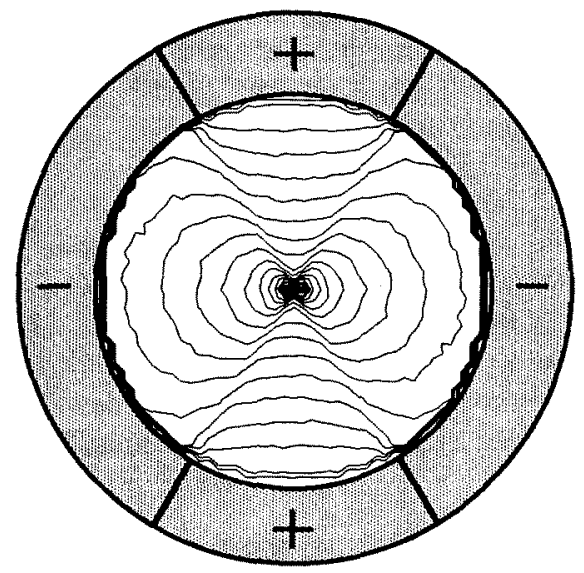

(a)

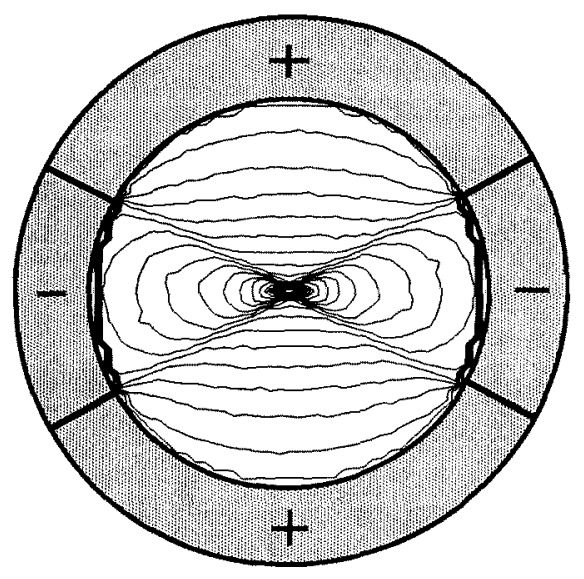

(b)

Fig. 10. The $S^{+}(x, y)$ spatial distribution of the ion production in the cross section of the segmented hollow cathode discharge, for (a) $C: A=2: 1$, (b) $C: A=1: 2$ cathode - anode surface ratios. The discharge parameters are: $V=600 \mathrm{~V}$ and $p=7.5 \mathrm{mbar}$. The contour step corresponds to 0.1 change in the $S^{+}(x, y)$ function.

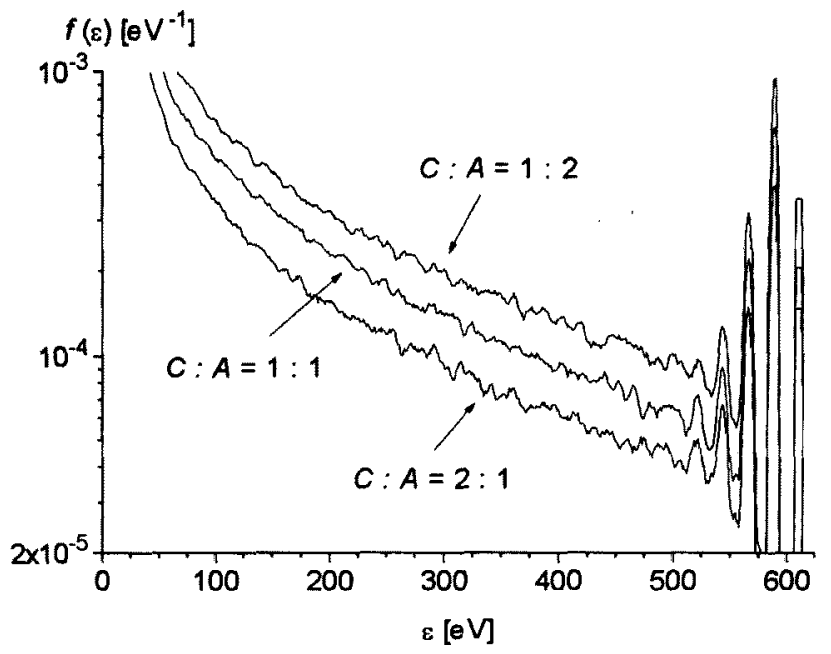

Fig. 11. The high energy part of the $f(\varepsilon)$ energy distribution of electrons absorbed by the anode in the segmented hollow cathode discharge, for $C: A=2: 1, C: A=1: 1$ and $C: A=1: 2$ cathode - anode surface ratios. The discharge parameters are: $V=600 \mathrm{~V}$ and $p=7.5 \mathrm{mbar}$.

Table 3. The effect of the cathode - anode surface ratio $(C: A)$ on $P_{0}$ and $\eta(V=600 \mathrm{~V}, p=7.5 \mathrm{mbar})$.

\begin{tabular}{c||c|c}
\hline$C: A$ & $P_{0}[\%]$ & $\eta[\%]$ \\
\hline \hline $2: 1$ & 17.5 & 17.1 \\
\hline $1: 1$ & 18.7 & 14.5 \\
\hline $1: 2$ & 20.2 & 11.4 \\
\hline
\end{tabular}

\section{Conclusions}

We have investigated the motion of electrons in a high voltage segmented hollow cathode (SHC) discharge. Using Monte Carlo simulation of the electrons' trajectories in the discharge, we have calculated discharges characteristics and studied the statistical properties of the electrons' motion. We have investigated discharges with four and six electrode segments (SHC -4 and SHC - 6), and characterized with 1:1 cathode - anode area. We have found that the ion production peaks sharply in the centre of the discharge, as a consequence of focusing the fast electrons by the curved cathode surfaces.

Our results have contributed to the understanding of the effect of longitudinal magnetic field on the SHC -4 and SHC -6 discharges. All the results summarized below are for the condition of fixed voltage.

We have found that in the case of the SHC -4 discharge: with increasing magnetic field (over the range of $0 m T$ to $20 m T$ ):

- the discharge current decreased (experiment, see Ref. 4)

- the number of high-energy electrons absorbed on the anode increased considerably,

- higher number of primary electrons were found, which were absorbed by the anode without making any ionization,

- the fraction of oscillating electrons decreased. 
In the case of the SHC -6 discharge : with increasing magnetic field (over the range of $0 \mathrm{mT}$ to $20 \mathrm{mT}$ ):

- the discharge current increased (experiment, see Ref. 4),

- the number of high energy electrons absorbed by the anode decreased,

- there was a lower number of primary electrons absorbed by the anode without making any ionization,

- the fraction of oscillating electrons increased.

The investigation of the changing cathode - anode surface ratio resulted in the following observations: the decreasing value of the C:A (cathode - anode) surface ratio was found to result in:

- a decreasing discharge current (experiment, see Refs. $7,8)$

- an increasing number of high energy electrons absorbed by the anode,

- increasing number of primary electrons absorbed by the anode without making any ionizations,

- decreasing number of oscillating electrons.

The comparison of the results of our studies and the experimentally observed discharge characteristics $(4,7,8)$ emphasizes the importance of the fast electrons and oscillating electrons and the discharge geometry in the determination of the discharge behaviour.

\section{Acknowledgments}

We thank L. Szalai for discussions on the subject of this work, and acknowledge the support of the Hungarian Science Foundation (OTKA Grants F-015502 and $\mathrm{T}-014909$ ) and the Australian Research Council.

\section{References}

(1) K.Rózsa, "Hollow cathode discharges for gas lasers" Z. Naturforschung vol. 35a, pp.649-664, 1980

(2) R.C.Tobin, K.A.Peard, G.Bode, K.Rózsa, Z.Donkó and L.Szalai, "High-gain hollow-cathode metal ion lasers for the UV and VUV" IEEE Journal of Selected Topics in Quantum Electronics vol.1, pp.830-836, 1995

(3) K.A.Peard, K.Rózsa and R.C.Tobin, "Parametric study of a high-voltage hollow-cathode infrared copper-ion laser" J. Phys. D: Appl. Phys. vol.27, pp.219-227, 1994

(4) K.A.Peard, Z.Donkó, K.Rózsa, L.Szalai and R.C.Tobin, "Comparison of $\mathrm{Cu}-\mathrm{II} 781 \mathrm{~nm}$ lasers using high-voltage hollow-cathode and hollowcathode-anode discharges" IEEE J. Quantum Electron. vol.30, pp.2157-2165, 1994

(5) K.A.Peard, R.C.Tobin, K.Rózsa and Z.Donkó, "A High-Voltage Hollow-Cathode Au-II 282-nm Laser" IEEE J. Quantum Electron. vol.30, pp.1181-1186, 1994

(6) Z.Zhang, N.D.Perry and R.C.Tobin, "Magnetic field enhanced performance of a copper hollow anode cathode laser" J.Applied Physics vol.71, pp.5338-5343, 1992

(7) B.Leigh, F.Robilliard, R.C.Tobin, K.Rózsa and Z.Donkó: "Dependence of $\mathrm{TEM}_{00}$ mode gain on cathode area in a metal ion laser", (Abstract) 10th Conference of the Australian Physical Society, 5-7 July 1995, Brisbane, Australia, p.103, 1995

(8) B.Leigh, R.C.Tobin, K.Rózsa and Z.Donkó: "Dependence of gain and output power on cathode to anode area ratio in a segmented hollow-cathode $\mathrm{Cu}$-II $781 \mathrm{~nm}$ laser", to be published

(9) Z.Donkó, K.Rózsa and R.C.Tobin, "Study of the electrons' motion in a high voltage segmented hollow cathode discharge" Proc. of the 11th International Conference on Gas Discharges and their Applications", Vol.II, pp.372-375, 11-15 September 1995, Chuo University, Kasuga, Bunkyo-ku, Tokyo, Japan, 1995

(10) Z.Donkó, K.Rózsa and R.C.Tobin, "Monte Carlo analysis of the electrons' motion in a high voltage segmented hollow cathode discharge" Journal of Physics D: Applied Physics vol.29, pp. 105-114, 1996

(11) J.P.Bouef and E.Marode, "A Monte Carlo analysis of an electron swarm in a non-uniform field: the cathode region of a glow discharge in helium" Journal of Physics D: Applied Physics vol.15, pp.2169-2187, 1982

(12) G.J.Fetzer and J.J.Rocca, "A Self-Consistent Model for Negative Glow Discharge Lasers: The Hollow Cathode Helium Mercury Laser" IEEE J. Quantum Electron. vol.28, pp.1941-1955, 1992

(13) F.J. de Heer and R.H.J.Jansen, "Total cross sections for electron scattering by He" J. Phys. B: At. Mol. Phys. vol.10, pp.3741-3758, 1977

(14) R.W.Labahn and J.Callaway, "Differential Cross Sections for the Elastic Scattering of $1-95 \mathrm{eV}$ Electrons from Helium" Phys. Rev. $A$ vol.2, pp.366-369, 1970

(15) R.Kollath, "Sekundärelektron-Emission fester Körper bei Bestrahlung mit Elektronen" in Encyclopaedia of Physics (Springer, Berlin) Vol.XXI. pp.232-301, 1956

(16) H.Helm, "Experimentelle Nachweis des PendelEffekts in einer niederdruck Hohlkathoden Entladung in Argon" $Z$. Naturforschung vol.27a, pp.1812-1820, 1972

(17) Z.Donkó, "A study of the Motion of High-Energy Electrons in a Helium Hollow Cathode Discharge" $Z$. Naturforschung vol.48a, pp.457-464, 1983

(18) V.I.Kolobov and L.D.Tsendin, "Analytic model of the hollow cathode effect" Plasma Sources Sci. Technol. vol.4, pp.551-560, 1995

(19) Z.Donkó, K.Rózsa, R.C.Tobin and K.A.Peard: "Modeling and measurements on an obstructed glow discharge in helium" Physical Review E vol.49, pp.3283-3289, 1994

(Manuscript received Pebruary 6, 1996. revised April 22, 1996) 


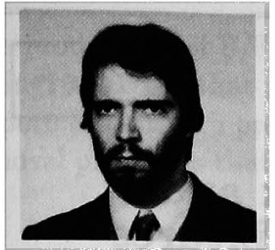

Zoltán Donkó (non-member) was born in Ózd, Hungary, in 1965. He was graduated and received the doctoral degree from the Technical University of Budapest in 1989 and 1992, respectively. Since 1992 he has been working at the Department of Laser Physics of the Research Institute for Solid State Physics, Budapest. His work is mainly concerned with basic research on glow discharges and hollow cathode lasers. For his research on the cathode region of glow discharges, in 1994 he was awarded with the Young Researchers' Prize of the Hungarian Academy of Sciences.

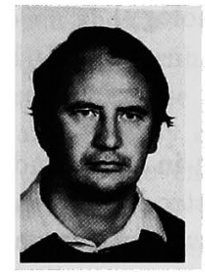

Károly Rózsa (non-member) was born in Budapest, Hungary, in 1939. He has been working for the Research Institute for Solid State Physics for gas laser and gas discharge research since 1963 . He graduated form the Technical University of Budapest as a chemical engineer and received his doctoral degree from laser spectroscopy. His main research work includes gas discharges, gas lasers, spectroscopy and photostimulated biological processes. Dr Rózsa is a member of IEEE, Lasers and Electro-Optics Society.

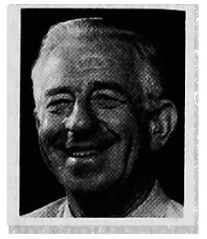

Roderick C. Tobin (non-member) was born in Melbourne, Australia, in September 1933. He received the B.Sc. (Hons.) degree from the University of Melbourne in 1960, and the Ph.D. degree from Monash University in 1980. After working as a Scientific Officer at the Aeronautical Research Laboratories, Melbourne from 1960 to 1964, he was appointed as a Lecturer in the Department of Physics, Monash University, in 1964, where he is currently a Reader. His main research interests are in the field of gas discharge lasers and particularly in sputtering-based metal vapour ultraviolet lasers. Dr Tobin is a Fellow of the Australian Institute of Physics and a Member of the Institute of Electrical and Electronics Engineers. 